\title{
Unexpected and fatal hemodynamic collapse during transapical transcatheter aortic valve implantation -A case report-
}

\author{
Eun Hee Kim ${ }^{1}$, Sangmin Maria Lee ${ }^{1}$, Jong-Hwan Lee ${ }^{1}$, Sang Hyun Lee ${ }^{1}$, Pyo Won Park ${ }^{2}$, and \\ Hyeon-Cheol Gwon ${ }^{3}$ \\ Departments of ${ }^{1}$ Anesthesiology and Pain Medicine, ${ }^{2}$ Thoracic and Cardiovascular Surgery, ${ }^{3}$ Cardiology, Samsung Medical Center, \\ Sungkyunkwan University School of Medicine, Seoul, Korea
}

Although transcatheter aortic valve implantation (TAVI) is generally accepted as an alternative or promising treatment option for patients with decompensated cardiovascular disease in an inoperable or high-risk condition, severe hypotension and/or arrhythmia associated with rapid ventricular pacing still poses a challenge to many clinicians. This report describes a 79-year-old patient who experienced fatal hemodynamic collapse, which suddenly developed after a rapid ventricular pacing in spite of pre-administration of vasopressor. The procedure and anesthesia were uneventful until the first rapid ventricular pacing was applied. Following rapid ventricular pacing, his cardiovascular state was severely compromised and could not be recovered. Despite early initiation of extracorporeal membrane oxygenation device and supportive care, he died from heart failure on post-procedure day four. (Korean J Anesthesiol 2013; 64: 360-362)

Key Words: Heart failure, Hemodynamic collapse, Rapid ventricular pacing, Transcatheter aortic valve implantation.

Since its first introduction in 2002 [1], transcatheter aortic valve implantation (TAVI) has been increasingly used in symptomatic patients with severe aortic stenosis [2]. Besides the well-known effectiveness in inoperable patients [3], many studies have suggested that TAVI can be a promising treatment option in high-risk patients [4-6] and associated with functional and hemodynamic improvement [3,7-10]. However, in spite of the recent favorable data, essential rapid ventricular pacing, followed by profound hypotension and arrhythmia during valvuloplasty and prosthesis implantation, is still a challenging problem to the anesthesiologists. In this report, we'd like to describe a case of unexpected and fatal hemodynamic collapse, which suddenly developed after a rapid ventricular pacing, despite achieving all currently-recommended anesthetic protocols.

Received: April 23, 2012. Revised: June 14, 2012. Accepted: June 18, 2012.

Corresponding author: Sangmin Maria Lee, M.D., Ph.D., Department of Anesthesiology and Pain Medicine, Samsung Medical Center, Sungkyunkwan University School of Medicine, 50, Ilwon-dong, Gangnam-gu, Seoul 135-710, Korea. Tel: 82-2-3410-0362, Fax: 82-2-3410-0361, E-mail: maria3run@gmail.com

(C)This is an open-access article distributed under the terms of the Creative Commons Attribution Non-Commercial License (http:// creativecommons.org/licenses/by-nc/3.0/), which permits unrestricted non-commercial use, distribution, and reproduction in any medium, provided the original work is properly cited. 


\section{Case Report}

A 79-year-old man was admitted with severe aortic stenosis. He suffered from chest discomfort on minimal exertion. His medical history included 5-times percutaneous coronary stent insertions for unstable angina, chronic obstructive pulmonary disease, atrial fibrillation, and atherosclerosis obliterans. Preprocedural echocardiography showed severe aortic stenosis (severe thickened and calcified aortic valve, aortic valve area $=0.72 \mathrm{~cm}^{2}$, aortic annulus $=22 \mathrm{~mm}$ ) and mild to moderate aortic regurgitation, moderate tricuspid regurgitation, both atrial enlargement, and concentric left ventricular hypertrophy. However, left ventricular systolic function (ejection fraction $=63 \%)$ and right ventricular systolic pressure $(28 \mathrm{mmHg}$ estimated by the maximal velocity of tricuspid regurgitation) were in normal range. The estimated logistic Euroscore of the patient was $29.8 \%$. The cardiologists recommended transapical TAVI instead of conventional surgery because the patient was considered as the high-risk population.

After placing an invasive arterial catheter through a brachial artery, anesthesia was induced with intravenous midazolam, etomidate, and rocuronium. It was maintained with sevoflurane and a continuous infusion of remifentanil. A pulmonary artery catheter was placed through right internal jugular vein, and a transesophageal echocargiographic (TEE) probe was inserted. After a left anterior mini-thoracotomy, an angiocatheter was inserted through the apex of the left ventricle. A guidewire was passed through the aortic valve and arch, and the tip was positioned in the abdominal aorta for stability. Following the two times of rapid ventricular pacing (200 beats $/ \mathrm{min}$ ) for 10 seconds, the balloon valvuloplasty was performed. Norepinephrine $(0.02-0.05 \mathrm{mcg} / \mathrm{kg} / \mathrm{min})$ was continuously infused for maintaining blood pressure and norepinephrine 5 mcg was successively injected twice prior to RVP for balloon valvuloplasty. From anesthetic induction to rapid ventricular pacing, the patient's vital signs were well maintained (systolic blood pressure, 90 to $144 \mathrm{mmHg}$; diastolic blood pressure, 46 to $70 \mathrm{mmHg}$; central venous pressure, 7 to $12 \mathrm{mmHg}$; mean pulmonary arterial pressure, 20 to $22 \mathrm{mmHg}$; and heart rate, 68 to 110 beats $/ \mathrm{min}$ ). In addition, the mean arterial pressure was also maintained at around $50 \mathrm{mmHg}$, during rapid ventricular pacing.

Following the balloon valvuloplasty with rapid ventricular pacing, the mean blood pressure dropped to below $40 \mathrm{mmHg}$ and did not recover. At the same time, central venous and the mean pulmonary arterial pressures were raised up to 22 $\mathrm{mmHg}$ and $44 \mathrm{mmHg}$, respectively. TEE showed the severe global hypokinesia. Two minutes after a rapid ventricular pacing, ventricular fibrillation was developed. Several attempts of defibrillation failed to restore the sinus rhythm. Emergent extracorporeal membrane oxygenation (ECMO) device was initiated immediately, through femoral artery and vein. Coronary angiography showed normal coronary arterial flow. After starting the support by ECMO, the patient's hemodynamics became stable. A 26-mm Sapien transcatheter heart valve (Edward Lifesciences, Irvine, CA, USA) was deployed under the ECMO support. TEE and angiography showed merely minimal aortic regurgitation. The patient was transferred to an intensive care unit for further supportive care. However, regardless of the ECMO support and supportive medical treatments, he did not recover from the state of heart failure. Finally, he died from cardiogenic shock and heart failure on post-procedure day 4 .

\section{Discussion}

In this mortality case, we experienced an unexpected event of fatal hemodynamic collapse, which developed immediately after a rapid ventricular pacing, although the recommended anesthetic managements were applied. Previously, there has been a case report that described the severity of cardiovascular compromise after a valve implantation due to the coronary arterial occlusion [11]. However, in our case, coronary arterial blood flow was normal.

Generally, for TAVI procedure, similar general anesthetic technique for high risk conventional aortic valve replacement is recommended [12]. In addition, bolus administration of a vasopressor prior to, or immediately after, a rapid ventricular pacing has been suggested to maintain coronary perfusion pressure $[2,12]$. Continuous monitoring of TEE is also strongly recommended, and a hybrid operating room, which is able to initiate cardiopulmonary bypass or to convert into conventional surgery, is considered as the ideal place for the TAVI procedure $[2,12]$. Those recommendations were all achieved in this case. Therefore, we'd like to evaluate the possible causes of this fatal event.

First, transapical approach might be potentially harmful to the heart because it involves the introduction of a large catheter through the ventricular apex. An inevitable introduction of large-bore catheter during transapical TAVI could cause myocardial damage, followed by arrhythmia $[5,13]$. Second, we focused on the vague point of recommended anesthetic protocols for TAVI. Although an administration of a bolus vasopressor is generally advocated, the amount of vasopressor should be titrated optimally in these patients because a relatively small dose would lead to insufficient coronary perfusion during a rapid ventricular pacing, and too much would cause severe elevation in the pulmonary arterial pressure $[14,15]$. In addition, an increase in pulmonary arterial pressure would worsen the right ventricular contractility and cardiac output 
$[14,15]$. However, to our knowledge, there is no recommendation for the individual optimal dose of vasopressor during a rapid ventricular pacing essential to the TAVI procedure. Moreover, the "optimal" dose of vasopressor would likely be different in patients because of the individual variation. Additionally, cardiac output is absent during rapid ventricular pacing. Without cardiac output, it would be questionable whether it is possible to predict the appropriate amount of vasopressor for the adequate coronary perfusion, as well as to preserve the coronary flow merely by maintaining the mean arterial pressure of around $50 \mathrm{mmHg}$.

In conclusion, the anesthesiologists should keep in mind that the fatal hemodynamic collapse can develop after a rapid ventricular pacing, despite the strict adherence to currentlyrecommended anesthetic protocols. In addition, further studies would be recommended to find the individual optimal dose of vasopressor for more successful anesthetic management of TAVI procedure.

\section{References}

1. Cribier A, Eltchaninoff H, Bash A, Borenstein N, Tron C, Bauer F, et al. Percutaneous transcatheter implantation of an aortic valve prosthesis for calcific aortic stenosis: first human case description. Circulation 2002; 106: 3006-8.

2. Covello RD, Landoni G, Zangrillo A. Anesthetic management of transcatheter aortic valve implantation. Curr Opin Anesthesiol 2011; 24: 417-25.

3. Leon MB, Smith CR, Mack M, Miller DC, Moses JW, Svensson LG, et al. Transcatheter aortic-valve implantation for aortic stenosis in patients who cannot undergo surgery. N Eng J Med 2010; 363: 1597607.

4. Tamburino C, Capodanno D, Ramondo A, Petronio AS, Ettori F, Santoro G, et al. Incidence and predictors of early and late mortality after transcatheter aortic valve implantation in 663 patients with severe aortic stenosis. Circulation 2011; 123: 299-308.

5. Rodes-Cabau J, Webb JG, Cheung A, Ye J, Dumont E, Feindel CM, et al. Transcatheter aortic valve implantation for the treatment of severe symptomatic aortic stenosis in patients at very high or prohibitive surgical risk: acute and late outcomes of the multicenter Canadian experience. J Am Coll Cardiol 2010; 55: 1080-90.

6. Godino C, Maisano F, Montorfano M, Latib A, Chieffo A, Michev I, et al. Outcomes after transcatheter aortic valve implantation with both Edwards-SAPIEN and CoreValve devices in a single center: the Milan experience. JACC Cardiovasc Interv 2010; 3: 1110-21.

7. Krane M, Deutsch MA, Bleiziffer S, Schneider L, Ruge H, Mazzitelli D, et al. Quality of life among patients undergoing tanscatheter aortic valve implantation. Am Heart J 2010; 160: 451-7.

8. Bekeredjian R, Krumsdorf U, Chorianopoulos E, Kallenbach K, Karck M, Katus HA, et al. Usefulness of percutaneous aortic valve implantation to improve quality of life in patients $>80$ years of age. Am J Cardiol 2010; 106: 1777-81.

9. Ewe SH, Ajmone Marsan N, Pepi M, Delgado V, Tamborini G, Muratori M, et al. Impact of left ventricular systolic function on clinical and echocardiographic outcomes following transcatheter aortic valve implantation for severe aortic stenosis. Am Heart J 2010; 160: 1113-20.

10. Guarracino F, Talini E, Landoni G, Petronio S, Giannini C, Di Bello $\mathrm{V}$. Effect of aortic valve surgery on left ventricular diastolic assessed by echocardiography and neuroendocrine response: percutaneous versus surgical approach. J Cardiothorac Vasc Anesth 2010; 24: 25-9.

11. Crimi G, Passerone G, Rubartelli P. Trans-apical aortic valve implantation complicated by left main occlusion. Catheter Cardiovasc Interv 2011; 78: 656-9.

12. Billings FT 4th, Kodali SK, Shanewise JS. Transcatheter aortic valve implantation: anesthetic considerations. Anesth Analg 2009; 108: 1453-62.

13. Rodes-Cabau J, Gutierrez M, Bagur R, De Larochelliere R, Doyle D, Cote $\mathrm{M}$, et al. Incidence, predictive factors, and prognostic value of myocardial injury following uncomplicated transcatheter aortic valve implantation. J Am Coll Cardiol 2011; 57: 1988-99.

14. Fischer LG, Van Aken H, Burkle H. Management of pulmonary hypertension: physiological and pharmacological considerations for anesthesiologists. Anesth Analg 2003; 96: 1603-16.

15. Price LC, Wort SJ, Finney SJ, Marino PS, Brett SJ. Pulmonary vascular and right ventricular dysfunction in adult critical care: current and emerging options for management: a systematic literature review. Crit Care 2010; 14: R169. 